\title{
Grain-Boundary Phase Transformation of ZnO:Pr System
}

\author{
Yukio Sato ${ }^{1}, J_{1}-Y o u n g ~$ Roh $^{1}$, and Yuichi Ikuhara ${ }^{1,2,3}$ \\ 1. Institute of Engineering Innovation, The University of Tokyo, Tokyo, Japan \\ 2. Nanostructures Research Laboratory, Japan Fine Ceramics Center, Nagoya, Japan \\ 3. World-Premier Institute, The Advanced Institute for Materials Research, Tohoku University, Sendai, \\ Japan
}

Grain boundary (GB) is two-dimensional lattice defect in crystals. Since the presence of GB often has a big impact on the physical properties, the atomic and electronic level structures have been intensitvely investigated. For making the atomic-scale structural analysis possible, GBs with well-defined orientation relationship are used for the analysis, which has been very succesful. With the invension of abbrationcorrector combined with scanning transmission electron microscopy, GB atomic structure as well as the location of segregation atoms can be routinely observed nowadays. However, since GB structure and segregation vary largely with the change of crystallographic orientation relationship and GB plane normal directions, there are so many possible structures in real materials. Although an ultimate goal of GB structure study is therefore to understand all different GBs with different geometries, it is quite unrealistic to study a large number of GBs with the atomic scale. For resolving this discrepancy, it is important to undrestand the relationship between the GB strcuture and geometry. In the present study, we aim to figure out the structural variation with the change of tilt angle $(\theta)$ in $\mathrm{ZnO}[0001]$ symmetric tilt GBs with the segregation of praseodymium (Pr). This system is widely utilized for varistor devices due to the highly nonlinear current-voltage characteristics.

Our previous studies have revealed the atomic structure of the Pr-doped $\mathrm{ZnO}$ [0001] $\Sigma 49$ and $\Sigma 7$ $\left(\theta=16.4^{\circ}\right.$ and $\left.21.8^{\circ}\right)$ GBs $[1,2]$. Here, the structure can be described with the structural unit (SU) model ( $\alpha$ and $\beta$ ) in Fig. 1). Owing to the benefit of $Z$-dependent contrast and incoherent imaging nature [3], the atomic structure is directly observed, and $\operatorname{Pr}(Z=59)$, which is much heavier than $\operatorname{Zn}(Z=30)$, is observed as brightest spots (Fig. 1). Our recent investigation toward higher $\theta$ reveals that SU changes into the different sort $(\alpha(\beta)$ to $\gamma)$ with $\theta$ close to $30^{\circ}$ (Fig. 2). This is different tendency for the undoped $\mathrm{ZnO}$ GB case, where the structural transition does not occur (SUs $\alpha$ and/or $\beta$ appear in the whole $\theta$ range). This suggests that Pr-doping induces the structural transition at the $\theta$ close to $30^{\circ}$, showing a coorperative chemical and geometrical effect to GB structure. Regarding SU as a "phase" for the GB, SU- $\theta$ diagram as in Fig. 2 may be called GB phase diagram. We expect that this would be useful for understanding the GB structure-geometry relationship. Further details will be given in the presentation.

\section{References:}

[1] Y. Sato, T. Mizoguchi, N. Shibata, T. Yamamoto, T. Hirayama, and Y. Ikuhara, Phys. Rev. B, 80, 094114 (2009).

[2] Y. Sato, J. P. Buban, T. Mizoguchi, N. Shibata, M. Yodogawa, T. Yamamoto, and Y. Ikuhara, Phys. Rev. Lett. 97, 106802 (2006).

[3] S. J. Pennycook and D. E. Jesson, Phys. Rev. Lett. 64, 938 (1990).

[4] Acknowledgements: A part of this work is supported by the Grants-in-Aids for Young Scientists (B) and Scientific Research on Priority Areas (474) from JSPS and MEXT, Nihon Sheet Glass Foundation for Materials Science and Engineering, and Kato Foundation for Promotion of Science. Theoretical 
works were done with supercomputer system in Institute of Solid State Physics (ISSP) of The University of Tokyo.

(a)

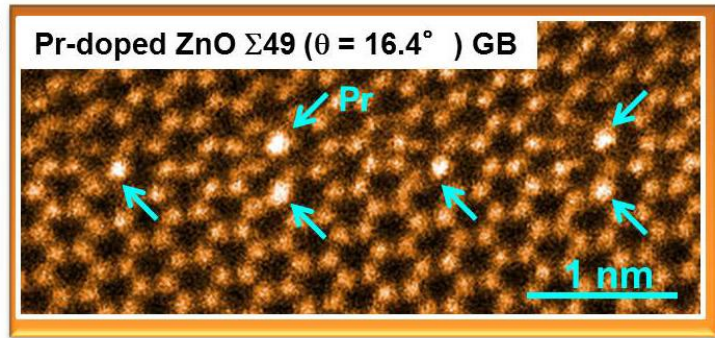

(b)

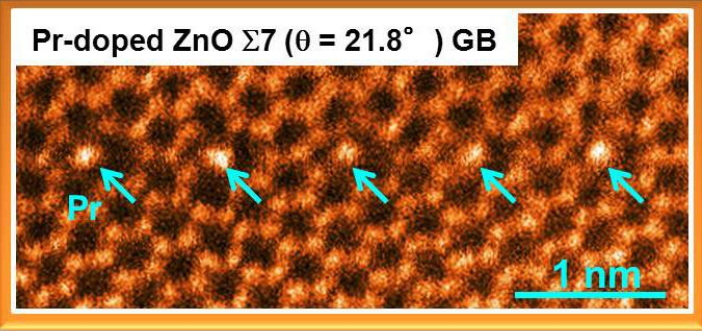

(c) Tilt angle $(\theta)$

$16.4^{\circ}(\Sigma 49)$

$21.8^{\circ}(\Sigma 7)$
Structural units

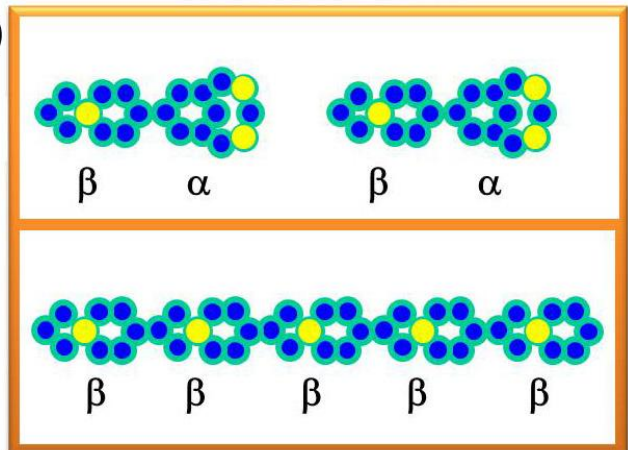

$\begin{array}{ll}\bigcirc & \mathrm{Zn} \\ \bigcirc & \mathrm{Pr} \\ & 0\end{array}$

Figure 1. Z-contrast STEM images of Pr-doped $\mathrm{ZnO}$ [0001] (a) $\Sigma 49$ and (b) $\Sigma 7\left(\theta=16.4^{\circ}\right.$ and $\left.21.8^{\circ}\right)$ GBs $[2,3]$. (c) Corresponding SUs are shown on the right-hand side.

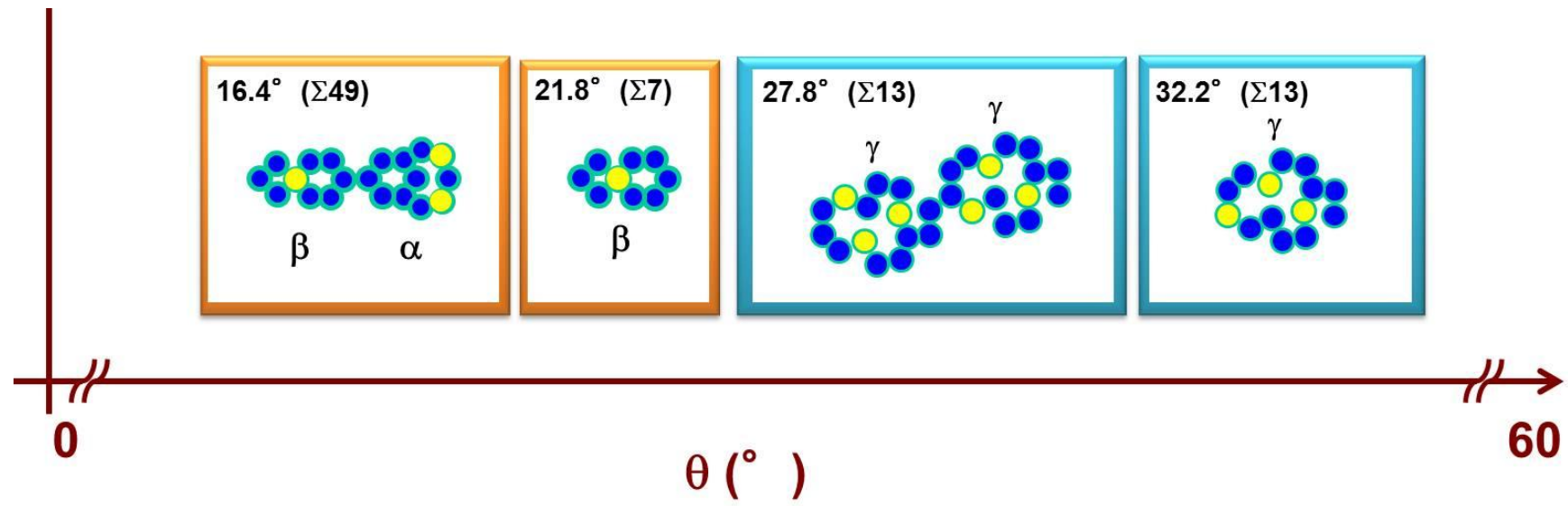

Figure 2. GB structure $-\theta$ diagram for the Pr-doped $\mathrm{ZnO}[0001]$ symmetric tilt GB system. 